\title{
Ibrutinib Regimens in Older Patients with Untreated CLL
}

TO THE EDITOR: Woyach et al. (Dec. 27 issue) ${ }^{1}$ report that in their trial involving patients who were 65 years of age or older with previously untreated chronic lymphocytic leukemia (CLL), treatment with ibrutinib was superior to bendamustine plus rituximab with regard to progression-free survival. The authors also report that the rate of grade 5 adverse events was higher than expected with the ibrutinib-containing regimens. They thought this finding might be due to the crossover design and short follow-up. However, in the Adverse Events section of the article, the authors mention that they excluded events that occurred after crossover, so I think this finding could not have been related to the crossover design.

The rate of grade 5 adverse events, especially unexplained or unwitnessed death, appears to be significantly higher in this trial than in similar trials. ${ }^{2,3}$ However, the rate of grade 5 adverse events other than unexplained or unwitnessed death was consistent with that in previous reports. I think this finding might be related to lethal ventricular tachyarrhythmias that may develop in patients who receive ibrutinib. ${ }^{4}$

Omer Diker, M.D.

Near East University Hospital

Nicosia, Cyprus

omeromrumdiker@gmail.com

No potential conflict of interest relevant to this letter was reported.

1. Woyach JA, Ruppert AS, Heerema NA, et al. Ibrutinib regimens versus chemoimmunotherapy in older patients with untreated CLL. N Engl J Med 2018;379:2517-28.

2. Moreno C, Greil R, Demirkan F, et al. Ibrutinib plus obinutuzumab versus chlorambucil plus obinutuzumab in first-line treatment of chronic lymphocytic leukaemia (iLLUMINATE): a multicentre, randomised, open-label, phase 3 trial. Lancet Oncol 2019;20:43-56.

3. Barr PM, Robak T, Owen C, et al. Sustained efficacy and detailed clinical follow-up of first-line ibrutinib treatment in older patients with chronic lymphocytic leukemia: extended phase 3 results from RESONATE-2. Haematologica 2018;103: 1502-10.

4. Lampson BL, Yu L, Glynn RJ, et al. Ventricular arrhythmias and sudden death in patients taking ibrutinib. Blood 2017;129: 2581-4.

DOI: 10.1056/NEJMc1901284

TO THE EDITOR: Woyach et al. make a sweeping conclusion regarding the superiority of ibrutinib over bendamustine plus rituximab in older pa- tients with CLL. Design flaws in the methods of the trial inherently lent a selection bias favoring ibrutinib by including patients with del(17p13.1) and TP53 mutations (who should not receive chemoimmunotherapy $)^{1}$ and by continuing ibrutinib until disease progression as compared with the 6 months of therapy with bendamustine plus rituximab. The lack of a between-group difference at year 1 in patients without del(17p13.1) and del(11q22.3) is testimony to this bias (Fig. S2 in the Supplementary Appendix, available with the full text of the article at NEJM.org). In addition, no improvement in outcomes was seen in patients with methylation at the promoter region of the gene encoding zeta chain-associated protein kinase 70 (ZAP70), who made up nearly half the patients (47\%) (Table S2 in the Supplementary Appendix).

The significant difference between ibrutinib and bendamustine plus rituximab with respect to progression-free survival at 2 years was thus driven largely by patients with these mutations. A tempering of conclusions is warranted, since the position of ibrutinib as a first-line drug is as yet unclear and it has a great cost and potential for serious toxic effects. ${ }^{2}$

Manju Sengar, M.D., D.M.

Tata Memorial Centre

Mumbai, India

manju.sengar@gmail.com

Nithya Gogtay, M.D., Ph.D.

King Edward Memorial Hospital

Mumbai, India

Hasmukh Jain, M.D., D.M.

Tata Memorial Centre

Mumbai, India

No potential conflict of interest relevant to this letter was reported.

1. Eichhorst B, Fink AM, Bahlo J, et al. First-line chemoimmunotherapy with bendamustine and rituximab versus fludarabine, cyclophosphamide, and rituximab in patients with advanced chronic lymphocytic leukaemia (CLL10): an international, openlabel, randomised, phase 3, non-inferiority trial. Lancet Oncol 2016;17:928-42.

2. Kater AP, Brown JR. Ibrutinib: searching for a partner drug. Lancet Oncol 2019;20:3-5.

DOI: $10.1056 / N E J M c 1901284$

TO THE EDITOR: In a randomized, phase 3 trial involving patients with previously untreated CLL, 
Woyach et al. found that treatment with continuous ibrutinib, either alone or combined with rituximab, was superior to six cycles of chemoimmunotherapy with regard to progression-free survival. However, the incidence of clinically significant nonhematologic adverse events was higher among the patients who received ibrutinibcontaining regimens.

Inhibition of Bruton's tyrosine kinase (BTK) by ibrutinib has emerged as a crucial strategy for treating B-cell cancers. However, BTK also plays a major role in the development and functioning of adaptive immunity, including fungal immune surveillance. Recent studies have shown higherthan-expected rates of life-threatening invasive fungal diseases among patients who have received ibrutinib. These diseases usually occur early in the course of treatment and manifest with nonclassic features. ${ }^{1,2}$ Table S7 in the Supplementary Appendix of the article by Woyach et al. shows a $19.1 \%$ rate of infection of grade 3 or higher (in 69 of 361 patients) among patients who received ibrutinib in the trial.

Could the authors provide additional information on the underlying species and clinical manifestations of these infections, especially with respect to fungal pathogens? Given the alarming incidence of invasive fungal diseases in some studies, ${ }^{3}$ we think that this aspect should be properly addressed in current and ongoing prospective multicenter trials. ${ }^{4}$

Toine Mercier, M.D.

Ann Janssens, Ph.D.

Johan Maertens, Ph.D.

Catholic University of Leuven

Leuven, Belgium

johan.maertens@uzleuven.be

Dr. Janssens reports receiving consulting fees, speaker's fees, and travel support from Janssen Pharmaceutica. No other potential conflict of interest relevant to this letter was reported.

1. Ahn IE, Jerussi T, Farooqui M, Tian X, Wiestner A, GeaBanacloche J. Atypical Pneumocystis jirovecii pneumonia in previously untreated patients with CLL on single-agent ibrutinib. Blood 2016;128:1940-3.

2. Ghez D, Calleja A, Protin C, et al. Early-onset invasive aspergillosis and other fungal infections in patients treated with ibrutinib. Blood 2018;131:1955-9.

3. Lionakis MS, Dunleavy K, Roschewski M, et al. Inhibition of $\mathrm{B}$ cell receptor signaling by ibrutinib in primary CNS lymphoma. Cancer Cell 2017;31(6):833-843.e5.

4. Chamilos G, Lionakis MS, Kontoyiannis DP. Call for action: invasive fungal infections associated with ibrutinib and other small molecule kinase inhibitors targeting immune signaling pathways. Clin Infect Dis 2018;66:140-8.

DOI: 10.1056/NEJMc1901284
THE AUTHORS REPLY: In reply to Diker: in our article, we meant that the similar overall survival among patients in the three groups may be due to the crossover design and relatively short followup for the overall survival end point. However, we do agree with the comment that the unexplained or unwitnessed deaths could have been related to the lethal ventricular arrhythmias that have been seen with ibrutinib treatment, and this is noted in the Discussion section of our article. This association has been reported by multiple groups, and the mechanism of arrhythmias and identification of at-risk patients are the subjects of active investigation.

We do not agree with Sengar et al. that there were methodologic flaws in our trial. The inclusion of patients with del(17p) or TP53 mutations was intentional, since the crossover design of the trial allowed all patients to receive ibrutinib at a time when this drug was not available outside a clinical trial. Figure 2B, and Figure S2C in the Supplementary Appendix, show that among patients without del(17p) or del(11q), ibrutinibcontaining regimens were superior to bendamustine plus rituximab with regard to progression-free survival. Sengar and colleagues are correct that at this time there does not appear to be a difference in progression-free survival among the treatment groups in patients with ZAP70methylated disease. However, the trial was not powered to detect differences within subgroups, so trends should be interpreted with caution. In addition, most patients with ZAP70-methylated disease remained in remission, so longer followup is needed before firm conclusions can be made for this group. The superiority of ibrutinib over bendamustine plus rituximab in terms of progression-free survival in the entire patient population is clear, and this trial, along with others, ${ }^{1,2}$ has shown that ibrutinib is more effective than chemotherapy or chemoimmunotherapy.

Mercier et al. raise an important point about the risk of invasive fungal infections with ibrutinib. In this trial, toxic effects were documented and graded according to the Common Terminology Criteria for Adverse Events, and the investigators could provide additional comments regarding these toxic effects. Therefore, we knew of specific infection types only if this information was volunteered by investigators at the sites. One patient was noted to have aspergillus pneu- 
monia, but no other cases of invasive fungal infections were reported. An analysis of adverse events in this trial, with data on the types and sites of infections, including fungal infections, is under way.

Jennifer A. Woyach, M.D.

Amy S. Ruppert, Ph.D.

Ohio State University

Columbus, $\mathrm{OH}$

jennifer.woyach@osumc.edu

Sumithra J. Mandrekar, Ph.D.

Mayo Clinic

Rochester, MN

Since publication of her article, Dr. Woyach reports receiving clinical trial support from Pharmacyclics, consulting fees from Pharmacyclics, and laboratory research support from Loxo Oncology. No further potential conflict of interest relevant to this letter was reported.

1. Burger JA, Tedeschi A, Barr PM, et al. Ibrutinib as initial therapy for patients with chronic lymphocytic leukemia. N Engl J Med 2015;373:2425-37.

2. Shanafelt TD, Wang V, Kay NE. A randomized phase III study of ibrutinib (PCI-32765)-based therapy vs. standard fludarabine, cyclophosphamide, and rituximab (FCR) chemoimmunotherapy in untreated younger patients with chronic lymphocytic leukemia (CLL): a trial of the ECOG-ACRIN Cancer Research Group (E1912). Blood 2018;132:LBA-4. abstract.

\section{DOI: $10.1056 /$ NEJMc1901284}

Correspondence Copyright (c) 2019 Massachusetts Medical Society.

\section{INSTRUCTIONS FOR LETTERS TO THE EDITOR}

Letters to the Editor are considered for publication, subject to editing and abridgment, provided they do not contain material that has been submitted or published elsewhere.

Letters accepted for publication will appear in print, on our website at NEJM.org, or both.

Please note the following:

- Letters in reference to a Journal article must not exceed 175 words (excluding references) and must be received within 3 weeks after publication of the article.

- Letters not related to a Journal article must not exceed 400 words.

- A letter can have no more than five references and one figure or table.

- A letter can be signed by no more than three authors.

- Financial associations or other possible conflicts of interest must be disclosed. Disclosures will be published with the letters. (For authors of Journal articles who are responding to letters, we will only publish new relevant relationships that have developed since publication of the article.)

- Include your full mailing address, telephone number, fax number, and email address with your letter.

- All letters must be submitted through our online submission system at NEJM.org.
Letters that do not adhere to these instructions will not be considered. We will notify you when we have made a decision about possible publication. Letters regarding a recent Journal article may be shared with the authors of that article. We are unable to provide prepublication proofs. Submission of a letter constitutes permission for the Massachusetts Medical Society, its licensees, and its assignees to use it in the Journal's various print and electronic publications and in collections, revisions, and any other form or medium.

\section{NOTICES}

Notices submitted for publication should contain a mailing address and telephone number of a contact person or department. We regret that we are unable to publish all notices received.

\section{TH INTERNATIONAL MUTANT P53 WORKSHOP \& P53 ISOFORMS}

The workshop will be held in Lyon, France, May 15-18.

Contact the Institute for Advanced Biosciences, Site Santé, Allée des Alpes, 38700 La Tronche, France; or call +33 (0)4 76 5494 63; or e-mail mutantp53.2019@gmail.com; or see https:// www.mutantp53workshop.com.

\section{ND INTERNATIONAL CONGRESS OF HYPERTENSION} IN CHILDREN AND ADOLESCENTS

The conference will be held in Warsaw, Poland, May 24-26. Contact Paragon Group, 18 Avenue Louis-Casai, 1209 Geneva, Switzerland; or call +41 (0) 225330 948; or fax +41 (0) 22 5802 953; or e-mail secretariat@htpaediatrics.com; or see http://htpaediatrics.com.

\section{AMERICAN SOCIETY OF TROPICAL MEDICINE AND HYGIENE}

The "Update Course in Clinical Tropical Medicine and Travelers' Health" will be offered in Toronto, June 12 and 13. It will be held in conjunction with the North American Refugee Health Conference. The "ASTMH 68th Annual Meeting" will be held in National Harbor, MD, Nov. 20-24.

Contact the American Society of Tropical Medicine and Hygiene, 241 18th St. South, Suite 501, Arlington, VA 22202; or call (571) 351-5409; or e-mail info@astmh.org; or see https:// www.astmh.org/annual-meeting.

\section{TH WORLD CONGRESS OF LYMPHOLOGY}

The conference, presented by the International Society of Lymphology, will be held in Buenos Aires and Iguazd, Argentina, Sept. 23-28.

Contact Arenales 3605 PB1, C1425BEW, Ciudad Autónoma de Buenos Aires, Argentina; or call +54 11 4832-2742; or e-mail info@lymphology2019.com; or see http://lymphology2019.com.

\section{MAYO CLINIC SCOTTSDALE}

The following course will be offered in Scottsdale, AZ: " 5 th Annual Gastroenterology and Hepatology Update and Interactive Live Endoscopy" (Oct. 4 and 5).

Contact Mayo School of Continuous Professional Development, Mayo Clinic, 13400 E. Shea Blvd., Scottsdale, AZ 85259; or call (480) 301-4580; or fax (480) 301-8323; or e-mail mca.cme@ mayo.edu; or see http://www.mayo.edu/cme. 